\title{
Le syndrome de la diarrhée récurrente chez le porc charcutier : approches écopathologique et microbiologique
}

\author{
A. JESTIN, F. MADEC et M.R. POPOFF * \\ Ministère de l'Agriculture, Direction de la Qualité, \\ Services vétérinaires, Station de Pathologie porcine, \\ Les Croix, B.P. 9, F 22440 Ploufragan \\ * Services des Anaérobies, Institut Pasteur \\ F 75724 Paris Cedex 15
}

\begin{abstract}
Résumé
Un nouveau type de pathologie digestive a été identifié et décrit chez le porc à l'engrais : la diarrhée récurrente. Une étude écopathologique a été menée dans 70 élevages et 300 informations concernant le logement et l'alimentation ont été collectées. Dans les conditions expérimentales, des contaminants associés aux diarrhées ont été isolés et leur pouvoir pathogène testé.

Les principales circonstances associées aux troubles se rapportent à la composition de l'aliment : la présence d'ingrédients comme le manioc, la mélasse, les petits pois, les graisses favorisent les diarrhées. Le Rotavirus, l'entérotoxine de $C$. perfringens et des protozoaires inoculés per $o s$ induisent les diarrhées. Le développement de ces dernières est principalement associé à certaines conditions d'élevage : un mode de distribution de l'aliment au sol, une mauvaise isolation thermique, une ventilation défectueuse et une faible surface de caillebotis au sol.
\end{abstract}

Mots clés : Porc, diarrhée, manioc, entérotoxine, protozoaires.

\section{Introduction}

Récemment, un nouveau syndrome diarrhéique, dénommé "Diarrhée récurrente " a été décrit en Bretagne (MADEc et al., 1984). Les troubles apparaissent généralement trois semaines environ après l'entrée des animaux dans la porcherie d'engraissement. Le plus souvent la diarrhée ne se déclare pas brutalement et, à des phases de simple ramollissement des fèces, font suite des épisodes de diarrhées franches.

Les coronavirus de la gastro-entérite transmissible et de la diarrhée épidémique porcine ou les bactéries (Treponema hyodysenteriae), agents étiologiques classiques des troubles digestifs du porc (Morin et al., 1973 ; VAnNier \& Debouck, 1983 ; TAYlor \& Alexander, 1971 ; Raynaud et al., 1980), n'ont pas été retrouvés dans ce syndrome.

Une enquête écopathologique a été mise en place dans les élevages afin de préciser les conditions favorisant l'apparition des troubles. Dans les conditions expérimentales, des animaux ont été infectés avec des contaminants isolés d'animaux malades afin de préciser leur rôle. 


\section{Matériel et méthode}

\section{A. Etude écopathologique}

\section{Protocole de l'enquête}

La collecte des données épidémiologiques est réalisée dans soixante-dix élevages à l'occasion d'une seule visite de l'enquêteur, à la sixième semaine après l'entrée des animaux en porcherie d'engraissement.

\section{Enregistrements}

Les points suivants donnent lieu à enregistrement :

a) Description des conditions de logement des animaux à contrôler

Les mensurations du local sont réalisées et reportées sur un plan. De même, les conditions bioclimatiques ainsi que les renseignements relatifs à la conduite du bâtiment sont enregistrés.

b) Observation du comportement des porcs et des manifestations cliniques

Les déjections sont examinées en détail par case et les données classées en 3 types (tabl. 1), dans l'ordre de gravité.

\section{TABLeau 1}

Répartition des élevages en fonction des troubles digestifs observés.

Classification of herds according to the digestive disorders.

\begin{tabular}{|c|c|c|}
\hline $\begin{array}{l}\text { Classe d'élevage } \\
\text { Herd classes }\end{array}$ & $\begin{array}{l}\text { Nombre d'élevages } \\
\text { Number of herds }\end{array}$ & $\begin{array}{l}\text { Symbole } \\
\text { Symbol }\end{array}$ \\
\hline \multicolumn{3}{|l|}{ Animaux - Animals } \\
\hline - sans problème - healthy & $17(24,2 \%)$ & OBJ 1 \\
\hline - déjections de consistance ramollie - soft faeces .. & $14(20 \%)$ & OBJ 2 \\
\hline — déjections diarrhéiques - diarrhoeic faeces . . . . & $39(55,8 \%)$ & OBJ 3 \\
\hline Total & $70(100 \%)$ & \\
\hline
\end{tabular}

\section{c) Alimentation}

Sont examinés les modes de distribution et de présentation (granulés ou farine), les quantités distribuées et la composition de la ration. Cette composition concerne aussi bien les matières alimentaires (composition centésimale) que les divers additifs et les thérapeutiques (nature, dose et durée d'application). 


\section{Réalisation des prises de sang}

Sur un échantillon de 10 porcs pour recherches sérologiques: anticorps de la gastro-entérite transmissible, diarrhée épidémique porcine, grippe, maladie d'Aujeszky.

\section{Traitement statistique des données}

Le nombre de renseignements collectés est d'environ 300 pour chacun des 70 élevages de l'étude. L'exploitation statistique d'un tel tableau a été réalisée à l'aide de la méthode de l'analyse des données (BEnzecri, 1976).

\section{a) Description élémentaire de l'échantillon}

Les éléments descriptifs (ou variables) sont envisagés séparément à l'aide des méthodes statistiques habituelles: histogrammes, paramètres de dispersion. Les tableaux de comptage et les calculs des coefficients de corrélation entre variables permettent de mettre en relief les premières relations existant pour des variables prises deux à deux.

b) Traitement proprement dit comportant deux étapes :

- la détermination de la typologie des élevages selon l'acuité des problèmes ;

- la recherche méthodique des circonstances associées aux troubles.

\section{B. Etudes microbiologiques au laboratoire}

\section{Animaux}

Quatre lots de cinq porcs sont placés dans des conditions d'isolement (salle étanche, filtration de l'air, douche pour le personnel...). Les animaux sont élevés par lots sur sol métallique ajouré (flat-deck) mais dans le but de se rapprocher des conditions de la pratique, un tapis de caoutchouc est mis en place et recouvre $50 \mathrm{p} .100$ de la surface au sol. L'eau est distribuée à volonté ainsi que l'aliment en granulés placés dans des auges ( 5 auges par case). Les animaux font l'objet des contrôles quotidiens suivants : l'allure générale, la température rectale, la consistance des excréments. Tous les jours, des échantillons d'excréments sont prélevés et immédiatement dirigés vers le laboratoire aux fins d'analyses. Une fois par semaine, les porcs sont pesés et les quantités d'aliment consommé relevées.

On a expérimenté un aliment dit «traditionnel » à base de céréales et un aliment dit « à risque » à l'égard des troubles digestifs, dont la composition se rapprochait le plus de celle des aliments que consommaient les animaux malades dans les élevages.

\section{Protocoles expérimentaux}

Deux expériences sont effectuées, afin d'étudier le rôle des protozoaires vivant dans le caecum et le côlon dans l'apparition de la diarrhée récurrente et de préciser l'influence de la composition de l'aliment sur l'évolution de cette diarrhée. 
a) Rôle des rotavirus, entérotoxine et protozoaires

Deux lots de 5 porcelets pesant $10 \mathrm{~kg}( \pm 1,1)$, obtenus par hystérectomie et élevés en conditions contrôlées reçoivent un aliment à risque (tabl. 2). Un premier lot placé en salle séparée, est infecté par voie orale, avec une suspension (10 10 germes) de culture d'une souche de Clostridium perfringens de type $\mathrm{A}$ isolée chez le porc et avec $40 \mathrm{ml}$ d'une suspension de rotavirus titré par Eusa (titre 1/810). Le virus a été obtenu par multiplication sur porcelets axéniques inoculés par des filtrats de contenus digestifs de porcs charcutiers infectés.

Le deuxième lot d'animaux a été contaminé dans les mêmes conditions, après avoir été infecté par une suspension de un million de kystes de Trichomonas suis et d'Entamoeba polecki produits par culture des trophozoïtes obtenus à partir de porcs atteints de diarrhées récurrentes.

\section{b) Etude de l'influence de la composition de l'aliment}

Dans une même animalerie, deux lots de 5 porcs SPF pesant en moyenne $15 \mathrm{~kg}$ $( \pm 2)$ sont placés dans deux loges grillagées différentes. Un lot consomme l'aliment à risque et un second l'aliment traditionnel. Tous les porcs sont contaminés par une suspension de fèces diarrhéiques prélevées sur les animaux du second lot de l'expérience précédente.

TABLEAU 2

Composition centésimale des aliments testés.

Composition of diets (\%).

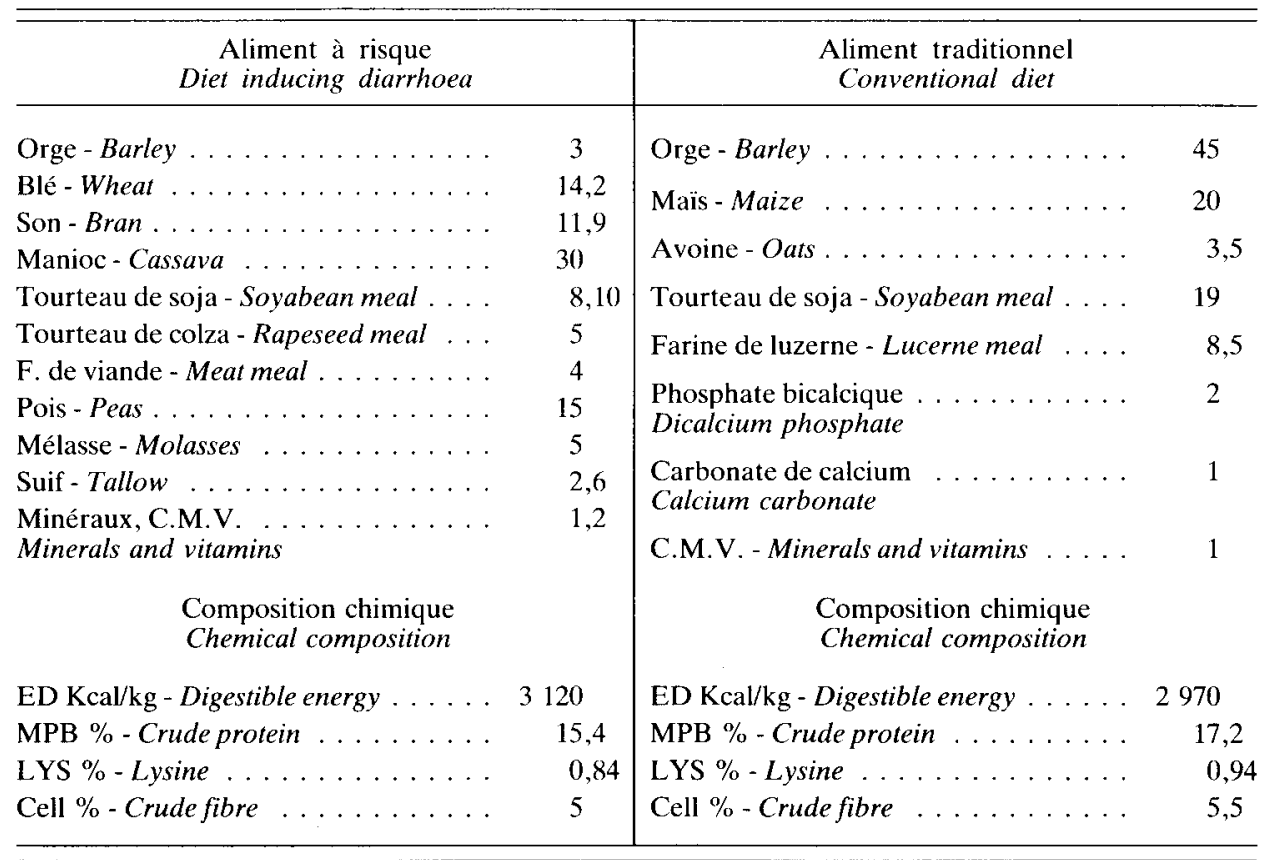




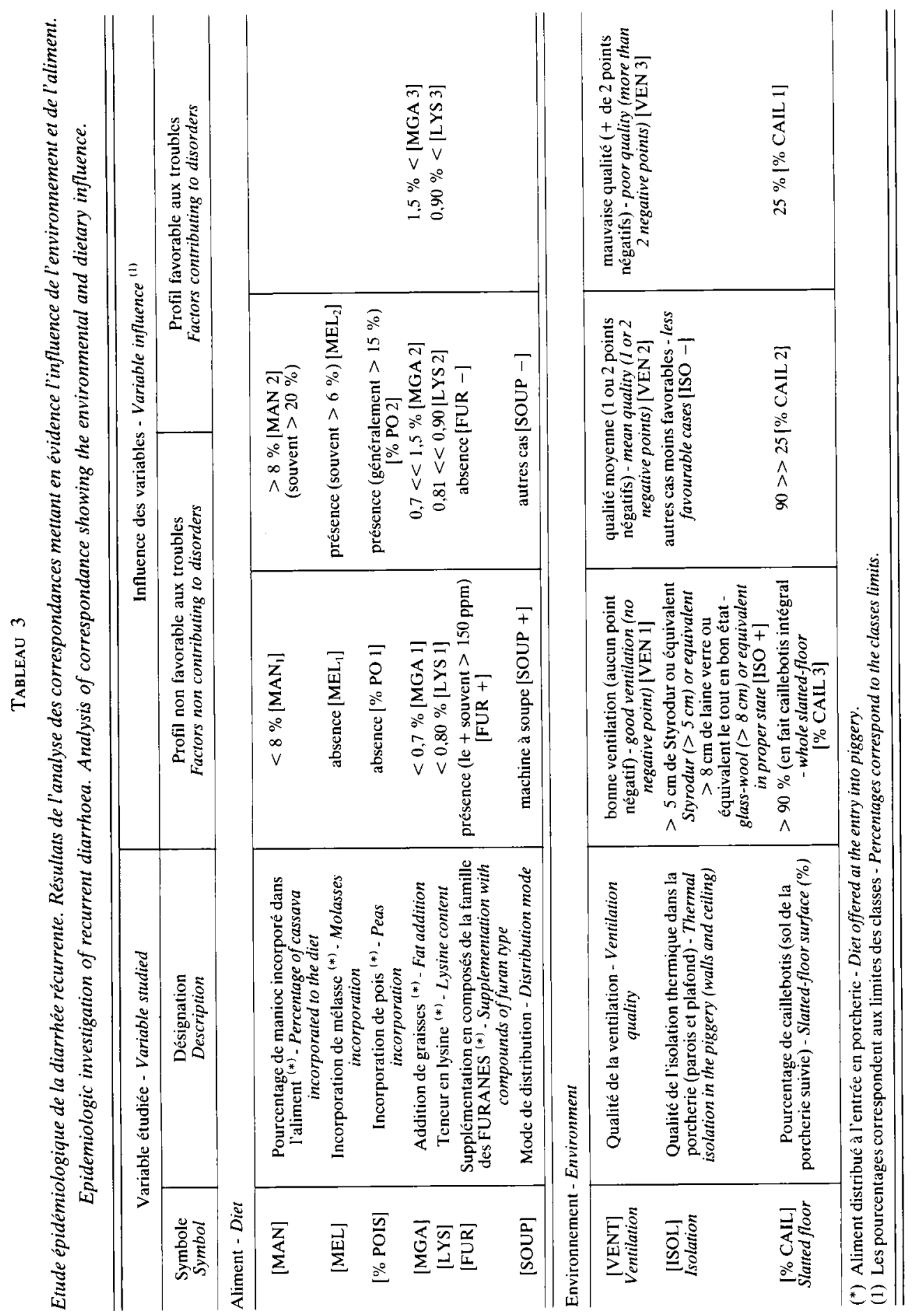




\section{Analyses de laboratoire}

L'entérotoxine dans les matières fécales est titrée par la technique de contre immuno électrophorèse (NAIK \& DUNCAN, 1977). Le Rotavirus est titré dans les fèces de porcs, également par la technique Elisa (Scherrer \& Bernard, 1977).

\section{Résultats}

\section{A. Etude écopathologique}

1. Circonstances associées au syndrome de la diarrhée récurrente dans les porcheries

L'enquête a permis de préciser les circonstances associées à l'apparition du syndrome de la diarrhée récurrente (tabl. 3). Sur les 10 variables favorables à son

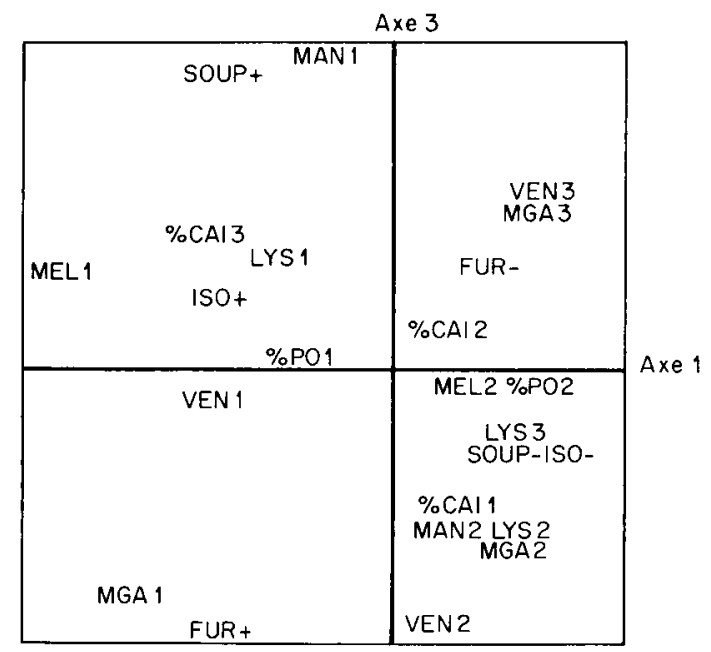

FIG. 1

Etude épidémiologique de la diarrhée récurrente par l'analyse factorielle des correspondances.

Position des variables.

Epidemiologic investigation of recurrent

diarrhoea using factorial analysis of correspondances. Variable location.

MAN2 : pourcentage de manioc ( $>20 \%$ ) - proportion of cassava

MEL2 : incorporation de mélasse - molasses incorporation

$\%$ PO2 : incorporation de petits pois - green peas incorporation

MGA3 : addition de graisses $(>1,5 \%)$ - fat addition

LYS3 : teneur en lysine $(>0,9 \%)$ - lysine content

FUR + : addition de furane - furane addition

SOUP + : distribution de l'aliment en soupe - distribution of the diet as soup

VEN1 : bonne ventilation - good ventilation

ISO + : bonne isolation - good isolation

$\%$ CAI1 : pourcentage de caillebotis $(>90 \%)$ - slatted-floor surface 
déclenchement, 5 se rapportent à la composition de l'aliment : incorporation de manioc, de mélasses, de matières grasses, de petits pois ou de lysine. Deux autres variables se rapportent, soit aux modalités de distribution (type liquide), soit à l'addition de certaines molécules anti-infectieuses (furanes).

\section{Position des variables sur la carte}

Les variables étudiées sont réparties sur une carte qui correspond au plan défini par les deux axes principaux dans l'analyse (axes 1 et 3). Une ventilation et une isolation défectueuses (VEN 3), (ISO -), l'absence de furanes (FUR -), l'incorporation de graisse (MGA 2), (MGA 3), de manioc (MAN 2), de mélasse (MEL 2) ou de pois (\% PO 2) sont des conditions qui prédisposent aux troubles. Ces éléments sont regroupés sur la partie droite de la carte (fig. 1).

Sur la partie gauche de la carte (fig. 1) est réuni un autre groupe de variables comprenant : la distribution en soupe (SOUP + ), le caillebotis intégral (\% CAI 3), la ventilation correcte et l'isolation thermique satisfaisante (VEN 1), (ISO +), l'absence de manioc, de mélasse et de graisses dans l'aliment : (MAN 1), (MEL 1) et (MGA 1) et enfin une supplémentation médicamenteuse à base de furanes (FUR + ). Ces éléments sont associés à la non apparition des troubles.

\section{Répartition des observations}

La figure 2 montre l'accumulation des porcheries affectées par la diarrhée dans la région droite (31 sur 39 ). En revanche, des porcheries indemmes se localisent sur la partie gauche (11 sur 17). Enfin, les élevages dans lesquels les déjections ont, au moment de l'enquête, une consistance ramollie sont presque confondus avec les porcheries à diarrhée.

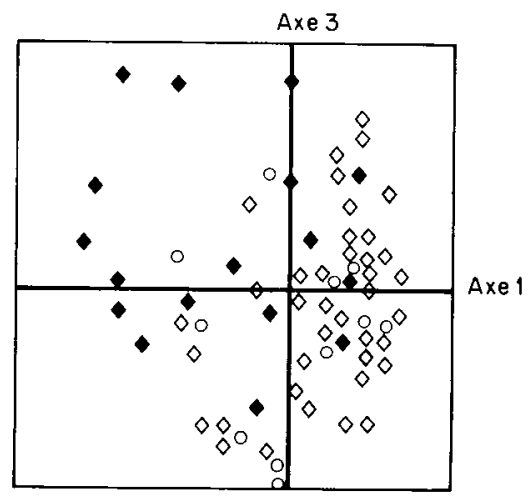

FIG. 2

Etude épidémiologique de la diarrhée récurrente par l'analyse factorielle des correspondances Position des élevages (FAC 1-3)

Epidemiologic investigation of recurrent diarrhoea using factorial analysis of correspondances. Herd location

$\checkmark$ porcheries à diarrhées [obj 3] - piggeries with diarrhoea

O porcheries à «bouses " [obj 2] - piggeries with cases of soft faeces

$\checkmark$ porcheries indemnes [obj 1] - piggeries without diarrhoea 
L'examen détaillé de la répartition des points montre l'existence de quatre porcheries indemnes au sein du groupe des porcheries à problèmes. Dans ces élevages, bien que toutes les conditions soient réunies pour l'apparition des troubles, les animaux ne sont pas malades.

\section{Répartition des élevages et positions des variables}

Les variables (MAN 1), (SOUP +), (\% CAI 3), (MEL 1), (ISO +), (VEN 1), (MGA 1), (FUR +), (LYS 1), (\% PO 1) sont situées sur la gauche de la carte. Leur localisation sur la zone occupée préférentiellement par des porcheries indemnes permet de les considérer comme variables défavorables à l'apparition des troubles (fig. 3).

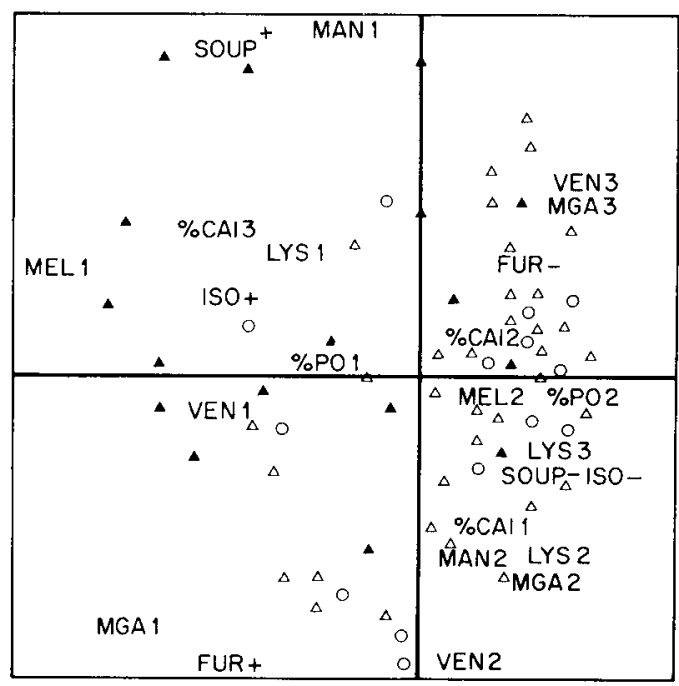

FIG. 3

Etude épidémiologique de la diarrhée récurrente: position relative des élevages et des variables étudiées

Epidemiologic investigation of recurrent diarrhoea

Relative location of herds and variables

$\triangle$ porcheries à diarrhées - piggeries with diarrhoea

0 porcheries à «bouses " - piggeries with cases of soft faeces

$\Delta$ porcheries indemnes - piggeries without diarrhoea

MAN2 : pourcentage de manioc $(>20 \%)$ - proportion of cassava

MEL2 : incorporation de mélasse - molasses incorporation

$\% \mathrm{PO} 2$ : incorporation de petit pois - green peas incorporation

MGA3 : addition de graisses $(>1,5 \%)$ - fat addition

LYS3 : teneur en lysine $(>0,9 \%)$ - lysine content

FUR + : addition de furane - furan addition

SOUP + : distribution de l'aliment en soupe - distribution of the diet as soup

VEN1 : bonne ventilation - good ventilation

ISO + : bonne isolation - good isolation

$\%$ CAI1 : pourcentage de caillebotis $(>90 \%)$ - slatted-floor surface 
(1)

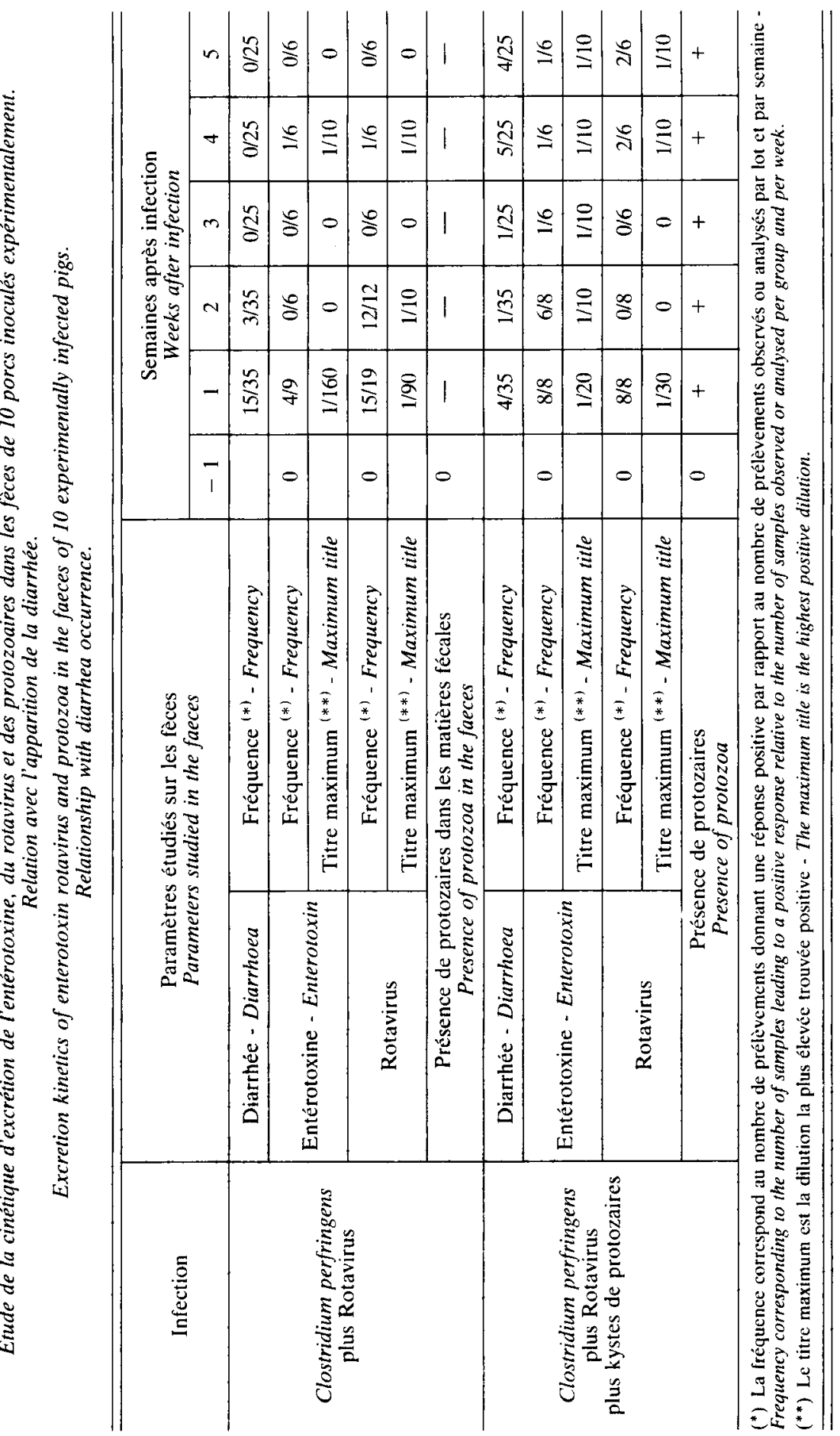


A l'opposé, les variables (MAN 2), (SOUP -), (\% CAI 1), (MEL 2), (ISO -), (VEN 2), (MGA 3), (FUR -), (LYS 3), (LYS 2), (\% PO 2) sont situées sur la zone correspondant aux porcheries où sont enregistrés des troubles digestifs. Ces résultats permettent de considérer ces variables comme prédisposantes à l'égard de ces troubles.

\section{B. Etudes microbiologiques à partir d'animaux infectés expérimentalement}

\section{Rôle des protozoaires}

Les diarrhées ont été observées dans les deux lots de porcs infectés (tabl. 4). L'infection par $C$. perfringens et le rotavirus est suivie par une excrétion de l'entérotoxine du Clostridium et du Rotavirus. L'infection par $C$. perfringens, le rotavirus et les kystes de protozoaires est également suivie par des diarrhées à double phase. La première dure une semaine et correspond, avec une intensité plus faible, au phénomène observé dans le lot précédent. La deuxième phase de diarrhée (récurrente) correspond à une récidive pendant la quatrième et la cinquième semaine de l'expérience.

L'entérotoxine est excrétée à des titres très élevés (1/160). Le Rotavirus est retrouvé avec une fréquence élevée (15/19 et 12/12) et à des titres importants (1/90) dans les diarrhées des animaux. Par la suite, les titres sont très faibles.

Pendant les troisième, quatrième et cinquième semaines, les animaux du lot infecté par les protozoaires présentent des diarrhées : à l'opposé, ceux qui ne sont pas infectés par ces protozoaires ne sont pas malades. Ces résultats sont en faveur du rôle déterminant des protozoaires dans l'évolution de la diarrhée récurrente chez les porcs excrétant l'entérotoxine et le rotavirus. La diarrhée est déclenchée par des agents actifs au niveau de l'intestin grêle; en effet, l'entérotoxine et le rotavirus ont pour site d'action l'iléon. L'évolution des troubles digestifs dépend de la présence des protozoaires dans le cæecum et le côlon de porcs dont la fonction iléale connaît de légères perturbations, consécutives à l'action discrète de l'entérotoxine et du virus.

\section{Influence de la composition de l'aliment}

Tous les porcelets infectés consommant un aliment à risque présentent des diarrhées. Par contre, les porcelets consommant un aliment traditionnel semblent indemnes (tabl. 5).

\section{TABLEAU 5}

Influence de la composition des aliments sur la fréquence des diarrhées. Effect of diet composition on diarrhoea frequency.

\begin{tabular}{c|c|c|c|c}
\hline $\begin{array}{c}\text { Aliment consommé (lot) } \\
\text { Ingested diet }\end{array}$ & \multicolumn{4}{|c}{$\begin{array}{c}\text { Semaines après l'infection } \\
\text { Weeks after infection }\end{array}$} \\
\cline { 2 - 5 } & 1 & 2 & 3 & 4 \\
\hline Aliment à risque $^{(*)}$ - Diet inducing diarrhoea ... & $5 / 35$ & $18 / 35$ & $12 / 35$ & $2 / 35$ \\
\hline Aliment traditionnel $^{(*)}$ - Conventional diet ..... & $0 / 35$ & $0 / 35$ & $0 / 35$ & $0 / 35$ \\
\hline
\end{tabular}

(*) La composition des aliments figure au tableau 2 - Diet composition is reported in table 2. 


\section{Discussion}

\section{A. Etude écopathologique}

Le choix préférentiel de porcheries à problèmes par les enquêteurs et l'incidence élevée des troubles dans les porcheries d'engraissement au cours de la période d'observation dans la région Ouest de la France explique la faible proportion de porcheries où les animaux sont indemnes. Dans les conditions d'enregistrements, les informations épidémiologiques collectées ne sont pas exhaustives.

Toutefois, la comparaison des conditions d'élevage et d'alimentation des porcs à l'engrais dans les différentes porcheries a permis d'identifier un groupe de circonstances plus particulièrement associées à la présence ou à l'absence des troubles. Parmi cellesci, la composition de l'aliment distribué aux animaux dès l'entrée en porcherie d'engraissement regroupe plusieurs facteurs de risque.

L'utilisation du manioc peut être associée à des conditions économiques pouvant expliquer des taux d'incorporation parfois élevés. Bien qu'on ne soit pas en mesure d'établir une relation directe entre la présence de ce composé et les troubles, le manioc apparaît cependant comme un élément favorisant le ramollissement des fèces, alors qu'à l'opposé son absence dans les formules, au profit des céréales, est un des éléments « de sécurité ». La même remarque peut être faite à propos d'autres matières premières alimentaires comme le pois fourrager, la mélasse et certaines graisses mais il est difficile de préciser l'effet de la qualité de ces matières premières. De plus, les résultats varient en fonction de l'âge de l'animal ; en effet, Aumaitre (1969) trouve une réduction de la diarrhée avec un bon manioc et HANSEN et SunEsen (1973) observent de tels troubles au-delà de $20 \%$ d'incorporation.

Les conditions de logement semblent également importantes dans le déterminisme des troubles. C'est le cas de la ventilation défectueuse, associée trop souvent à une mauvaise isolation thermique, ou même à un défaut de propreté de la loge. Cette situation favorise une contamination permanente du tube digestif par des germes de l'environnement, notamment lorsque l'alimentation est distribuée au sol, ce qui n'est pas possible en présence de caillebotis intégral.

\section{B. Etudes microbiologiques}

\section{Rôle de l'entérotoxine de $\mathrm{C}$. perfringens et du rotavirus}

La maladie est reproductible dans les conditions expérimentales. Les contaminants (C. perfrigens entérotoxinogènes et rotavirus) peuvent déclencher une diarrhée qui peut durer pendant plus de 10 jours. L'entérotoxine est retrouvée dans les cas de diarrhées récurrentes à des titres supérieurs à ceux retrouvés dans les selles humaines lors d'intoxication alimentaire (Popoff \& Jestin, 1985 ; SkJelkvale \& Uemura, 1977). Les rotavirus peuvent se multiplier au niveau de l'intestin grêle et perturber enfin les équilibres hydriques dans le sens de l'hydratation du contenu digestif.

\section{Rôle des protozoaires}

La présence de protozoaires au niveau du cæcum et du côlon semble conditionner l'évolution de troubles digestifs. Les perturbations au niveau de l'iléon entraînent le 
passage dans le cæcum de digesta qui modifient la composition physicochimique du contenu du cæcum. Ces modifications permettent l'expression du pouvoir pathogène des protozoaires qui sont responsables du phénomène de récurrence. La difficulté technique de séparation des kystes des protozoaires (Trichomonas et amibe) constitue un obstacle à la détermination précise du pouvoir pathogène respectif de ces agents.

\section{Influence de la composition de l'aliment}

Le second essai a mis en évidence le rôle déterminant de la composition de l'aliment dans l'expression des symptômes cliniques. Ces résultats peuvent être en relation avec des phénomènes pouvant avoir leur siège, soit au niveau de l'intestin grêle, soit au niveau du gros intestin.

L'incorporation du manioc dans l'aliment peut entraîner une désorganisation de la motricité au niveau de l'iléon (LAPLACE \& DARCY, 1984). L'excès d'apport de polysaccharides dans la ration peut entraîner une dysbactériose qui est suivie par une diarrhée (Weijers \& VAN DE KAMER, 1965). Le rôle des glucides du milieu a été défini dans la sporulation des C. perfringens. Un excès d'apport de polysaccharides entraînant la sporulation des bactéries serait favorable à la production d'entérotoxine (SACKs, 1983).

Les conditions de milieu qui permettent la multiplication des protozoaires ont fait l'objet d'études spécifiques; ainsi, la présence d'amidon non digéré conditionne la pullulation des protozoaires dans le cæcum du porc, en relation avec les bactéries du milieu (Schumaker, 1931). Si ces travaux permettent d'expliquer l'influence de la composition de l'aliment sur la multiplication des protozoaires, peu de données existent sur les conditions d'expression de leur pouvoir pathogène.

L'action du manioc peut être due à la présence de certains facteurs toxiques ou à la pollution microbiologique (Porsson et al. 1980).

L'incorporation à des taux élevés de graisses dans l'alimentation du porcelet n'affecte pas la digestibilité des autres composants de l'aliment et les essais effectués par Eusebio et al. (1965) n'établissent pas de relation entre les régimes riches en graisses et l'apparition de troubles digestifs.

En conclusion, l'analyse comparative des facteurs du milieu a souligné la nécessité de réunir un ensemble de conditions permettant l'expression du pouvoir pathogène de contaminants du tube digestif présents dans la majorité des élevages.

Il existe un type de diarrhées, dues aux protozoaires du cacum et du côlon, diarrhées qui ne sont rencontrées que chez les animaux dont la fonction iléale est perturbée par certains microbes (Rotavirus et $C$. perfringens). Ces diarrhées dites d'origines cæcale et colique sont une affection actuellement fréquente dans l'élevage du porc. Elles ont été signalées chez le porc à l'engrais sous le nom de diarrhée récurrente ; chez le porcelet sevré, une pathologie dite diarrhée grise présente de nombreuses analogies avec cette diarrhée récurrente. 


\section{Remerciements}

Les auteurs remercient A. Aumartre pour la correction du manuscrit et renouvellent leurs remerciements à $M^{\mathrm{me}}$ LE MENEC, Service de Bactériologie, Laboratoire Départemental d'Analyses, 22000 Saint-Brieuc, pour sa collaboration.

Reçu en juillet 1986.

Accepté en Janvier 1987.

\section{Summary \\ Ecopathologic and epidemiologic investigation of a diarrhoeic syndrome in bacon pigs}

A new diarrhoeic syndrome called "recurrent diarrhoea » was observed in pigs approximately three weeks after they were entered into the fattening unit. An ecopathologic investigation was performed in 70 herds and a total of 300 data about feeding and housing conditions were collected. The infectious agents associated to diarrhoeic were isolated and their pathogenicity tested.

Dietary components such as cassava, molasses, green peas and fat were found to contribute to diarrhoeic syndrome. The rotavirus, the $C$. perfringens enterotoxin and protozoa inoculated per os reproduced diarrhoea. The development of diarrhoea was mainly associated with herd management conditions : feed distribution on the floor, bad thermal isolation and ventilation, restricted surface of slatted floor.

Key words : Pig, diarrhoea, cassava, enterotoxin, protozoa.

\section{Références bibliographiques}

Aumaitre A., 1969. Valeur alimentaire du manioc et de différentes céréales dans les régimes de sevrage précoce du porcelet : utilisation digestive de l'aliment et effet sur la croissance des animaux. Ann. Zootech., 18, 385-398.

BenzeCRI J.P., 1976. L'analyse des données tome II. L'analyse des correspondances. Ed. Dunod, Paris. $616 \mathrm{p}$.

Eusebio J.A., Hays V.W., Speer V.C., Mc Call J.T., 1965. Utilization of fat by young pigs. $J$. Anim. Sci., 24, 1001-1007.

Hansen V., Sunesen N., 1973. Tapiokamel. Saertryk af Forsoegslab. Arbog 50-57 (en danois).

Laplace J.P., Darcy B., 1984, Motricité de l'intestin grêle et digestibilité iléale d'un régime à base de manioc chez le porc. Ann. Zootech., 33, 489-508.

MAdec F., Jestin A., Josse J., 1984. Le syndrome de la diarrhée récurrente dans les porcheries d'engraissement. I - Approche épidémiologique. Bull. Lab. Vét., 15, 13-28.

Morin M., Morehouse L. G., Solorzana R.F., Olson L.D., 1973. Transmissible gastro-enteritis in feeder swine: clinical, immunofluorescence and histopathological observations. Can. $J$. Comp. Méd., 37, 239-248.

NaIK H. S., DunCan C.L., 1977. Rapid detection and quantitation of Clostridium perfringens enterotoxin by counter immunoelectrophoresis. Appl. Environ. Microbiol., 34, 125-128. 
Poisson J., Drouet H., Gosset H., Vega D., 1980. Microflore des céréales et du manioc. Perspectives agricoles, 41, 40-55.

POPOFF M.R., JESTIN A., 1985. Enteropathogenicity of purified Clostridium perfringens enterotoxin in the pig: Am. J. Vet. Res., 46, 2147-2148.

Raynaud J.P., Brunault G., Philippe J., 1980. Swine dysentery. Comparison of experimental diseases produced by infection with colonic mucosa or with Treponema hyodysenteriae, French strains, and of "natural" disease. Ann. Rech. Vet., 11, 69-87.

SACKS L.E., 1983. Influence of carbohydrates on growth and sporulation of $C$. perfringens in a defined medium with or without guanosine. App. Environ. Microbiol., 46, 1169-1175.

Scherrer R., Bernard S., 1977. Application d'une technique immunoenzymologique (Elisa) à la détection du Rotavirus bovin et des anticorps dirigés contre lui. Ann. Microb., 128, 499-510.

SchUMAKER, 1931. Relation of Balantidium coli infection to the diet and intestinal flora of the domestic pig. Am. J. Hyg., 13, 576-582.

Skjelkvale R., Uemura T., 1977. Experimental diarrhoea in common volunteers following oral administration of Clostridium perfringens enterotoxin. J. Appl. Bacteriol, 43, 281-286.

TAYlor D.J., Alexander T.J.L., 1971. The production of dysentery in swine by feeding cultures containing spirochaetes. Br. Vet. J., 127, 58-61.

Vannier P., Debouck P., 1983. Identification de la diarrhée épidémique porcine en Bretagne : étude clinique. Rec. Méd. Vét., 159, 19-24.

Weijers H.A., VAN de KAmer J.H., 1965. Alteration of intestinal bacterial flora as a cause of diarrhoea. Nut. Abst. Rev., 35, 591-604. 